\section{Aleitamento materno no estado de Pernambuco: prevalência e possível papel das ações de saúde}

\section{Breastfeeding in the state of Pernambuco: prevalence and possible role in the health care}

\author{
Liliane de Jesus Bittencourt 1 \\ Juliana Souza Oliveira 2 \\ José Natal Figueiroa 3 \\ Malaquias Batista Filho 4
}

\begin{abstract}
Objectives: to describe prevalence, modalities and duration of the breast feeding, and possible factors of protection of exclusive breast feeding $(E B F)$.

Methods: probabilistic sample of 602 children details of 18 months, extracted of a data base of 2075 children details five years studied in its domiciles of 20 cities of the State of Pernambuco; study casecontrol of 24 children that had used exclusively brest milk up to four months of age, compared with 72 controls weaned before two months; analyze of logistic regression for factors of protection of the EBF.

Results: 24 children (10.75\%) had used the EBF up to four months, prevalence that declined for $3.87 \%$ to the six months. In the bivaried analyses, they had appeared as protection factors: schooling maternal, income, precocious beginning and number of prenatal consultations, surgical childbirth, medical attendance in the childbirth and use of private services of health. In the final model they had remained significant: income, beginning prenatal and distance of health services.

Conclusions: the duration of EBF was well lesser that the results of other studies. The mothers who had more suckled its children for drawn out periods distinguish for the income highest, beginning of prenatal and distance of health services.
\end{abstract}

Key words Breast feeding, Prevalence

\author{
Coordenação de nutrição. Coordenação de saúde. Faculdade de \\ Tecnologia e Ciência. Salvador, BA, Brasil. \\ 2 Departamento de Nutrição. Centro de Ciências da Saúde. \\ Universidade Federal de Pernambuco, Recife, Brasil. \\ 3,4 Departamento de Pesquisa. Instituto Materno Infantil Prof \\ Fernando Figueira, IMIP. Rua dos Coelhos, 300. Recife, PE, Brasil. \\ CEP: $50.070-550$
}

\section{Resumo}

Objetivos: descrever a prevalência, modalidades e duração do aleitamento materno e os fatores de proteção da amamentação exclusiva.

Métodos: amostra probabilística de 602 crianças menores de 18 meses, extraída de um banco de dados de 2075 crianças menores cinco anos, estudadas em seus domicílios de 20 municípios do estado de Pernambuco; estudo caso-controle de 24 crianças que usaram exclusivamente o leite materno até quatro meses de idade, comparadas com 72 controles totalmente desmamadas antes de dois meses de vida; análise de regressão logística para fatores de proteção ao aleitamento materno exclusivo (AME).

Resultados: apenas 24 crianças (10,75\%) utilizaram o AME até quatro meses, prevalência que declinou para 3,87\% aos seis meses. Nas análises bivariadas, apareceram como fatores de proteção: escolaridade da mãe, renda, início precoce (primeiro trimestre) e número de consultas pré-natais, parto cirúrgico, atendimento médico no parto e utilização de serviços privados de saúde. No modelo final permaneceram significativas: a renda, o início do pré-natal e a proximidade dos serviços de saúde.

Conclusões: a duração do AME foi bem menor que os resultados de outros estudos. As mães que amamentaram seus filhos por períodos mais prolongados se distinguem pela renda mais elevada, início do pré-natal e distância dos serviços de saúde.

Palavras-chave Aleitamento materno, Prevalência 


\section{Introdução}

Dois eventos são de fundamental importância para o prognóstico da sobrevivência, do crescimento e desenvolvimento saudável da criança nos seus primeiros meses e anos de vida: o peso ao nascer e os padrões de aleitamento materno.1,2 Universais em sua influência, essas condições são particularmente relevantes nos chamados países e regiões pobres, onde os fatores de riscos ambientais e socioeconômicos operam com freqüência, intensidade e interação mais adversa no processo saúde/doença, traduzindo-se em elevadas taxas de morbi-mortalidade, atraso de crescimento e outros handicaps que, inclusive, se manifestam em etapas bem posteriores do ciclo de vida: maturidade e senescência. ${ }^{3}$

As duas condições (baixo peso ao nascer e, notadamente, o desmame precoce) podem ser, em grande parte, corrigidas ou significativamente atenuadas mediante ações do setor saúde, no decurso da assistência materno-infantil (pré-natal, parto e cuidados pós-natais junto à mãe, o lactente e sua família). Interessa, neste artigo, a questão das práticas alimentares da criança no pós-nascimento imediato e nos primeiros meses de vida, tendo em vista os efeitos protetores do aleitamento materno na saúde infantil, evidenciado em estudos bem conclusivos em nível internacional 4 e, de forma bem consistente, no Brasil. Assim, Fuchs e Victora5 demonstraram que o risco relativo de morte em crianças, por conta das diarréias, infecções respiratórias agudas e outras doenças infecciosas, foi mais de 20 vezes mais elevado nas crianças precocemente desmamadas. Na realidade, o consenso estabelecido em nível internacional, sobre o papel protetor do aleitamento materno, justifica a meta das Nações Unidas, estabelecida na década passada, no sentido de que fossem oferecidas condições para que todas as mulheres do mundo pudessem amamentar seus filhos, de forma exclusiva, nos seis primeiros meses de vida,6,7 prolongando a amamentação, combinada com outras práticas alimentares, até o segundo ano de vida. Trata-se de um compromisso desafiador, tendo em conta a constatação da prática cosmopolita do desmame precoce e as tendências de sua consolidação, no mundo economicamente globalizado. 8

Nessas circunstâncias, se estabelece um contexto de marcantes antagonismos: de um lado, os interesses mercantilistas e a própria imagem dos valores impostos pela sociedade de consumo e suas demandas; de outro, o movimento de idéias e propostas ordenadas em função do interesse público, que se consolida na recomendação de resgatar as práticas da alimentação saudável, tendo como paradigma mais representativo a própria alimentação ao seio materno. A década de 90 , sobretudo em função das posições políticas e programáticas das Nações Unidas, tornou-se um período de experiências e mudanças expressivas, no processo de coexistência desses antagonismos. 9

$\mathrm{Na}$ realidade, os resultados em nível mundial e nacional são ainda inseguros: ao lado de avanços (20\% de aumento estimado nos índices de aleitamento materno até 2001), com resultados conflitivos nos Estados Unidos 10 na América Latina11 e bem modestos (18\%) nos países menos subdesenvolvidos. 12

No Brasil, a prevalência do aleitamento materno exclusivo aos quatro meses teria aumentado 10 vezes, entre 1986 e 1996.13 No entanto, em relação às metas das Nações Unidas, os resultados ainda se encontravam bem distantes dos padrões para o final da década (1999), visto que o aleitamento materno exclusivo só era praticado por $53,1 \%$ das mães aos 30 dias de vida de seus filhos, caindo para $9,7 \%$ aos seis meses. $14,15 \mathrm{Na}$ mesma ocasião, tomando como pontos de avaliação os 30 primeiros dias e os primeiros seis meses de vida, no Nordeste Brasileiro, os valores referentes ao AME eram, respectivamente, $55,4 \%$ e $10,7 \%$, portanto, bem aproximados dos resultados de âmbito nacional.

O presente estudo tem como objetivo avaliar a situação do aleitamento materno e suas modalidades no Estado de Pernambuco, construindo uma linha de base (1997) a partir da qual possam ser verificadas suas tendências nos diferentes estratos socioeconômicos, características da família e das mães, acesso e uso dos serviços de saúde. Partindo de famílias sorteadas em seus domicílios, corrige-se a tendência das investigações concentradas em áreas metropolitanas ou grandes cidades ou em clientelas dos serviços de saúde. Ao mesmo tempo, são investigados os possíveis fatores de proteção do aleitamento materno, com ênfase no papel da assistência à saúde, que constitui, em princípio, a vanguarda estratégica a ser mobilizada para a promoção do aleitamento materno. Por fim, o estudo, realizado com conceitos e metodologias padronizadas de coleta e análise de dados, torna-se pertinente, oportuno e até imprescindível para estabelecer comparações com um novo survey sobre a situação alimentar, nutricional e de saúde no Estado, a ser realizado em 2006. 


\section{Métodos}

Os dados utilizados neste estudo são oriundos do banco de informações coletadas na Segunda Pesquisa Estadual de Saúde e Nutrição.16 Nesse inquérito, o segundo realizado em Pernambuco, Brasil, na década de 90, foram estudadas famílias residentes em 1431 domicílios, compreendendo um total de 7748 pessoas. A amostra foi selecionada num processo de três estágios: a) no primeiro, foram sorteados 20 municípios, segundo um critério de "quase proporcionalidade" à sua população. A cidade do Recife, por exemplo, teve três chances de ser incluída no sorteio, em função de sua participação relativa na composição demográfica do Estado; b) no segundo estágio, foram escolhidos, por sorteio, os setores censitários (definidos pela Fundação Instituto Brasileiro de Geografia e Estatística, IBGE), dentro de cada município; c) numa terceira fase, foram sorteadas as famílias dentro de cada setor, com a condição preliminar de que contassem na sua composição com, pelo menos, uma criança menor de cinco anos (caso índice). Em cada setor deveriam ser estudadas em média, 50 crianças (de 45 a 55 "índices"). Mediante tal procedimento, foram identificados 514 domicílios (734 crianças) na Região Metropolitana do Recife, 490 domicílios (687 crianças) no Interior Urbano e, finalmente 427 municípios (654 crianças) no Interior Rural. O estudo compreendeu um conjunto de oito formulários (1-Identificação da família; 2-Registros Clínico/Laboratorial; 3-Registros do Domicílio; 4-Registros da Criança; 5-Registros de Morbidade; 6-Registros da Mulher; 7-Consumo alimentar da criança; 8-Consumo alimentar da família).

Os dados de campo foram obtidos por uma equipe de 12 entrevistadores (nutricionistas, biólogos, sociólogos, psicólogos, técnicos de laboratório) num trabalho de quase cinco meses, após a realização de um projeto piloto de 30 dias (teste dos questionários e capacitação do pessoal).

Em função dos objetivos específicos do presente estudo, foi gerado um banco ad hoc de dados, compreendendo apenas as crianças menores de 18 meses, o que permite o acompanhamento do processo de aleitamento materno em seu histórico mais importante, evitando-se, ademais, o chamado "viés de memória" que resultaria na anamnese dos antecedentes de crianças mais velhas.

As características do aleitamento materno (variável dependente) foram classificadas em três categorias: a) aleitamento materno exclusivo (AME), quando a criança apenas mamava, sem incluir o uso de água ou de chás como bebida eventual ou rotineira; b) aleitamento materno (AM), quando a criança, simultaneamente, recebia outros alimentos, sólidos, pastosos ou líquidos, incluindo o uso de água ou infusões; c) aleitamento interrompido (AI) a partir do momento em que a criança deixava, completamente, de efetuar qualquer mamada.

Como variáveis independentes, as características bio-sociais maternas (idade, número de filhos, estado nutricional segundo o índice de massa corporal (IMC), ocupação, renda per capita familiar), condições do pré-natal e do parto, história de assistência à saúde e dados ambientais (geográficos e habitacionais).

Os resultados foram apresentados em termos descritivos (evolução das práticas de aleitamento materno por meses de vida das crianças) e em nível analítico, considerando-se as hipóteses de associação de uma categoria de interesse específico e universal (o aleitamento materno exclusivo) com possíveis fatores de proteção, mediante o método caso/controle. Dada a raridade de sua ocorrência no limite mínimo de quatro meses (apenas 24 casos, no total da amostra de 603 crianças), essas situações foram assumidas como casos, em contraste com os controles (crianças que foram completamente desmamadas antes dos dois meses de vida). Para estimar as razões de chance, foram consideradas como referenciais as variáveis ou condições que, hipoteticamente, deveriam promover a duração do aleitamento materno exclusivo. As decisões estatísticas foram tomadas comparando-se as razões de chance (odds ratio), com um valor de $p$ (erro alfa) menor de 0,05 . Para cada caso foram tomados três controles.

Utilizou-se, ademais, a análise de regressão logística para descartar o possível efeito de variáveis de confusão e/ou papéis de colinearidade nos diversos resultados estatisticamente significativos. ${ }^{17}$ 


\section{Resultados}

Como se descreve na Tabela 1, até um mês de idade, $31,3 \%$ das crianças se alimentavam exclusivamente de leite materno. Aos seis meses, apenas seis crianças $(3,87 \%)$ faziam uso exclusivo do leite materno. Por outro lado, aos 30 dias de vida, 12,6\% das crianças já tinham sido completamente desmamadas, incluindo alguns casos que jamais chegaram a mamar. Na coluna que descreve o aleitamento materno de forma mista (somatório do AME mais a alimentação mista: leite materno conjugado com outra alternativa alimentar) verifica-se que, aos seis meses, apenas $1 / 4(25,7 \%)$ das crianças ainda mamavam, ao mesmo tempo em que se fazia uso de outras fontes alimentares. Aos 18 meses, apenas uma criança $(0,16 \%)$ ainda mamava.

No estudo caso/controle verificou-se que as vaiáveis biológicas referentes às mães (idade, número de filhos e estado nutricional) não se correlacionaram estatisticamente, com a ocorrência do aleitamento materno exclusivo mais prolongado (quatro meses ou mais) ou com o desmame mais prematuro (menos de dois meses de idade). Entre as chamadas variáveis sociais, a escolaridade e a renda mais elevadas atuaram como fatores de proteção ao aleitamento materno exclusivo. Das 11 mães de nível universitário, 7 (63,6\%) amamentaram seus filhos de forma exclusiva até os quatro meses, enquanto entre as 18 analfabetas, apenas $4(22,2 \%)$ atingiram este limite de duração do aleitamento materno exclusivo. A exposição à faixa salarial mais baixa (menos de um salário mínimo per capita) representou um handicap estatisticamente bem discriminante da duração do aleitamento materno. $\mathrm{O}$ fato de exercer ou não um trabalho profissional não se associa ao risco de desmame precoce (Tabela 2).

As condições de assistência pré-natal e ao parto (início dos exames pré-natais no primeiro trimestre de gravidez, realização de seis ou mais consultas pré-natais, o parto cirúrgico e o parto assistido pelo médico) atuaram como fatores de proteção em relação ao aleitamento materno. Já o fato de receber ou não orientação educativa sobre a amamentação não se correlacionou estatisticamente com o desfecho mais ou menos prolongado do processo de amamentação. Observa-se que apenas uma mãe, entre os 23 casos, e 10 entre os 55 controles não foram orientadas sobre condutas referentes à amamentação. O peso da criança ao nascer não atuou como um fator de diferenciação estatisticamente significativo em relação à duração do aleitamento materno.

\section{Tabela 1}

Aleitamento materno em crianças menores de 18 meses, no Estado de Pernambuco, Brasil, 1997.

\begin{tabular}{|c|c|c|c|c|c|c|}
\hline \multirow[t]{2}{*}{ Idade (meses) } & \multicolumn{2}{|c|}{ Aleitamento Materno Exclusivo(AME) } & \multicolumn{2}{|c|}{ Aleitamento Materno(AM) } & \multicolumn{2}{|c|}{ Aleitamento Interrompido(Al) } \\
\hline & $\mathrm{n}$ & $\%$ & $\mathrm{n}$ & $\%$ & $\mathrm{n}$ & $\%$ \\
\hline 1 & 165 & 31,3 & 527 & 87,39 & 76 & 12,6 \\
\hline 2 & 98 & 23,67 & 414 & 68,65 & 189 & 31,34 \\
\hline 3 & 56 & 18,12 & 309 & 51,24 & 294 & 48,75 \\
\hline 4 & 24 & 10,76 & 223 & 36,98 & 380 & 63,01 \\
\hline 5 & 11 & 6,04 & 182 & 30,18 & 421 & 69,81 \\
\hline 6 & 6 & 3,87 & 155 & 25,7 & 448 & 74,29 \\
\hline 12 & 0 & 0 & 44 & 7,29 & 559 & 92,7 \\
\hline 18 & 0 & 0 & 1 & 0,16 & 602 & 99,83 \\
\hline
\end{tabular}




\begin{tabular}{|c|c|c|c|c|c|c|c|c|}
\hline \multirow[t]{2}{*}{ Variáveis } & \multicolumn{2}{|c|}{$A M E \geq 4$ meses } & \multicolumn{2}{|c|}{ Desmame $<2$ meses } & \multirow[t]{2}{*}{ Total } & \multirow[t]{2}{*}{ OR } & \multirow[t]{2}{*}{ IC95\% } & \multirow[t]{2}{*}{$p$} \\
\hline & $\mathrm{n}$ & $\%$ & $\mathrm{n}$ & $\%$ & & & & \\
\hline \multicolumn{9}{|l|}{ Idade materna } \\
\hline $10-19$ anos & 2 & 8,3 & 7 & 9,7 & 9 & 0,76 & $0,09-4,80$ & 1 \\
\hline $20-29$ anos & 14 & 58,3 & 37 & 51,4 & 51 & 1 & & \\
\hline$>30$ anos & 8 & 33,3 & 28 & 38,9 & 36 & 0,76 & & \\
\hline Total & 24 & 99,9 & 72 & 100 & 96 & & & \\
\hline \multicolumn{9}{|l|}{ Estado nutricional } \\
\hline Baixo peso & 1 & 4,5 & 5 & 7 & 6 & 0,56 & $0,02-5,76$ & 1 \\
\hline Eutrófico & 15 & 68,2 & 42 & 59,2 & 57 & 1 & & \\
\hline Sobrepeso & 6 & 27,3 & 24 & 33,9 & 30 & 0,7 & $0,21-2,28$ & 0,513 \\
\hline Total & 22 & 100 & 71 & 100 & 93 & & & \\
\hline \multicolumn{9}{|l|}{ Número de filhos vivos } \\
\hline$>2$ & 9 & 37,5 & 26 & 37,1 & 35 & 1,02 & $0,335-2,92$ & 0,975 \\
\hline$\leq 2$ & 15 & 62,5 & 44 & 62,9 & 39 & 1 & & \\
\hline Total & 24 & 100 & 70 & 100 & 94 & & & \\
\hline \multicolumn{9}{|l|}{ Escolaridade } \\
\hline Não alfabetizada & 4 & 16,7 & 14 & 19,4 & 18 & 0,16 & $0,03-0,86$ & 0,032 \\
\hline Nível fundamental & 8 & 33,3 & 46 & 63,9 & 54 & 0,1 & $0,20-0,42$ & 0,002 \\
\hline Nível médio & 5 & 20,8 & 8 & 11,1 & 13 & 0,36 & $0,07-1,88$ & 0,224 \\
\hline Nível universitário & 7 & 29,2 & 4 & 5,6 & 11 & 1 & & \\
\hline Total & 24 & 100 & 72 & 100 & 96 & & & \\
\hline \multicolumn{9}{|l|}{ Ocupação } \\
\hline Trabalha & 10 & 41,7 & 19 & 26,4 & 29 & 1,99 & $0,68-5,82$ & 0,158 \\
\hline Não trabalha & 14 & 58,3 & 53 & 73,6 & 67 & 1 & & \\
\hline Total & 24 & 100 & 72 & 100 & 96 & & & \\
\hline \multicolumn{9}{|l|}{ Renda per capita $(\mathrm{SM})+$} \\
\hline$<1$ & 11 & 45,8 & 58 & 81,7 & 69 & 0,19 & $0,07-0,52$ & 0,001 \\
\hline$\geq 1$ & 13 & 54,2 & 13 & 18,3 & 26 & 1 & & \\
\hline Total & 24 & 100 & 71 & 100 & 95 & & & \\
\hline
\end{tabular}

AME = aleitamento materno exclusivo; SM: salário mínimo em $1997=\mathrm{R} \$ 120,00$ 
Na Tabela 4 se analisa o possível papel de outros aspectos da assistência à saúde na resposta sobre a duração mais prolongada do aleitamento materno exclusivo ou, inversamente, na instalação do desmame precoce, A visita regular do agente de saúde não se correlacionou estatisticamente com o desfecho da amamentação nos casos e controles, já o tipo de serviço utilizado (público x plano de saúde ou particular) evidencia uma diferenciação estatística significativa, aparecendo a alternativa "plano de saúde/particular" como uma condição que favorece a prática do aleitamento materno exclusivo.

Tabela 3

Condições do pré-natal e parto em relação ao aleitamento materno exclusivo. Pernambuco, 1997.

\begin{tabular}{|c|c|c|c|c|c|c|c|c|}
\hline \multirow[t]{2}{*}{ Variáveis } & \multicolumn{2}{|c|}{$A M E \geq 4$ meses } & \multicolumn{2}{|c|}{ Desmame $<2$ meses } & \multirow[t]{2}{*}{ Total } & \multirow[t]{2}{*}{ OR } & \multirow[t]{2}{*}{ IC95\% } & \multirow[t]{2}{*}{$p$} \\
\hline & $\mathrm{n}$ & $\%$ & $\mathrm{n}$ & $\%$ & & & & \\
\hline \multicolumn{9}{|l|}{ Início do pré-natal } \\
\hline $2^{\circ}$ e $3^{\circ}$ trimestre & 2 & 8,3 & 22 & 40 & 24 & 0,14 & $0,03-0,64$ & 0,011 \\
\hline $1^{\circ}$ trimestre & 22 & 91,7 & 33 & 60 & 55 & 1 & & \\
\hline Total & 24 & 100 & 55 & 100 & 79 & & & \\
\hline \multicolumn{9}{|c|}{ Número de consultas } \\
\hline$<6$ & 4 & 16,7 & 22 & 40,7 & 26 & 0,29 & $0,09-0,97$ & 0,044 \\
\hline$\geq 6$ & 20 & 83,3 & 32 & 59,3 & 52 & 1 & & \\
\hline Total & 24 & 100 & 54 & 100 & 78 & & & \\
\hline \multicolumn{9}{|c|}{ Orientação sobre aleitamento materno } \\
\hline Não & 1 & 4,2 & 10 & 18,2 & 11 & 0,2 & $0,01-1,66$ & 0,158 \\
\hline Sim & 23 & 95,8 & 45 & 81,8 & 68 & 1 & & \\
\hline Total & 24 & 100 & 55 & 100 & 79 & & & \\
\hline \multicolumn{9}{|l|}{ Tipo de parto } \\
\hline Cesariana & 14 & 58,3 & 16 & 22,2 & 30 & 4,9 & $1,83-13,10$ & 0,002 \\
\hline Normal & 10 & 41,7 & 56 & 77,8 & 66 & 1 & & \\
\hline Total & 24 & 100 & 72 & 100 & 96 & & & 0,001 \\
\hline \multicolumn{9}{|c|}{ Profissional que assistiu o parto } \\
\hline Não médico & 2 & 8,7 & 32 & 45,7 & 34 & 0,11 & $0,02-0,52$ & 0,005 \\
\hline Médico & 21 & 91,3 & 38 & 54,3 & 59 & 1 & & \\
\hline Total & 23 & 100 & 70 & 100 & 93 & & & \\
\hline \multicolumn{9}{|c|}{ Peso ao nascer (gramas) } \\
\hline$<2500$ & 1 & 4,2 & 8 & 12,1 & 9 & 0,32 & $0,01-2,76$ & 0,435 \\
\hline$\geq 2500$ & 23 & 95,8 & 58 & 87,9 & 81 & 1 & & \\
\hline Total & 24 & 100 & 66 & 100 & 90 & & & \\
\hline
\end{tabular}


Utilizando-se as oito variáveis que, analisadas em relação aos casos de amamentação exclusiva até o quarto mês de vida resultaram em associações estatisticamente significativas, procedeu-se a aplicação de uma análise multivariada (regressão logística) de modo a descartar os possíveis efeitos de simples colinearidade ou de variáveis de confusão. No modelo final, apresentado na Tabela 5, permaneceram como significativas a distância entre a residência e o serviço de saúde ( $>1 \mathrm{~km})$, o início do pré-natal no primeiro trimestre de gravidez e a renda per capita igual ou superior a um salário mínimo.

Tabela 4

História e condições de assistência à saúde em relação ao aleitamento materno exclusivo, Pernambuco, 1997.

\begin{tabular}{|c|c|c|c|c|c|c|c|c|}
\hline \multirow[t]{2}{*}{ Variáveis } & \multicolumn{2}{|c|}{$A M E \geq 4$ meses } & \multicolumn{2}{|c|}{ Desmame $<2$ meses } & \multirow[t]{2}{*}{ Total } & \multirow[t]{2}{*}{ OR } & \multirow[t]{2}{*}{$\mathrm{IC} 95 \%$} & \multirow[t]{2}{*}{$p$} \\
\hline & $\mathrm{n}$ & $\%$ & $\mathrm{n}$ & $\%$ & & & & \\
\hline \multicolumn{9}{|c|}{ Visita regular do agente de saúde } \\
\hline Não & 15 & 62,5 & 40 & 55,6 & 55 & 1,33 & $0,47-3,83$ & 0,551 \\
\hline $\operatorname{Sim}$ & 9 & 37,5 & 32 & 44,4 & 41 & 1 & & \\
\hline Total & 24 & 100 & 72 & 100 & 96 & & & \\
\hline \multicolumn{9}{|l|}{ Serviço de saúde utilizado } \\
\hline Público & 5 & 31,3 & 33 & 82,5 & 38 & 0,1 & $0,02-0,37$ & 0,001 \\
\hline Plano de saúde/ particular & 11 & 68,8 & 7 & 17,5 & 18 & 1 & & \\
\hline Total & 24 & 100,1 & 40 & 100 & 56 & & & \\
\hline \multicolumn{9}{|l|}{ Distância do serviço de saúde } \\
\hline$>1 \mathrm{~km}$ & 9 & 42,9 & 15 & 22,1 & 24 & 2,65 & $0,83-8,46$ & 0,06 \\
\hline$\leq 1 \mathrm{~km}$ & 12 & 57,1 & 53 & 77,9 & 65 & 1 & & \\
\hline Total & 21 & 100 & 68 & 100 & 89 & & & \\
\hline
\end{tabular}

Tabela 5

Fatores associados ao aleitamento materno exclusivo aos quatro meses de idade, segundo regressão logística.

Pernambuco, 1997.

\begin{tabular}{|c|c|c|c|c|}
\hline \multicolumn{5}{|c|}{ Distância do serviço em saúde } \\
\hline$\leq 1 \mathrm{~km}$ & 1 & 1 & & \\
\hline $2^{\circ}$ e $3^{\circ}$ trimestre & 0,14 & 0,06 & $0,01-0,45$ & 0,005 \\
\hline $1^{\circ}$ trimestre & 1 & 1 & & \\
\hline \multicolumn{5}{|l|}{ Renda per capita+ } \\
\hline
\end{tabular}

* Os dados referem-se ao OR ajustado; sm = salário mínimo; salário mínimo em 1997 = R\$120,00 


\section{Discussão}

A rápida substituição dos hábitos milenares e universais da amamentação na segunda metade do século passado, com a massificação industrial e mercantil do leite pasteurizado ou pulverizado, constitui, num curto espaço de tempo, a mudança mais radical nos padrões alimentares da humanidade em toda a sua história. É notável, ao mesmo tempo, a observação de que, rapidamente, foram sendo acumuladas evidências e comprovações científicas sobre as conseqüências adversas dessa mudança em vários aspectos do processo saúde/doença em relação à criança, à mãe, à família e sobre a própria coletividade: as várias dimensões biológicas, epidemiológicas, psicológicas, econômicas, sociais e até mesmo ecológicas do desmame precoce.

Dessa forma, a progressão generalizada da prática do desmame nos primeiros 25 anos que se seguiram aos anos 50 (século XX) passou a ser contraposta pelo movimento de resgate da amamentação no último quarto do século, mobilizando o poder público, instituições não-governamentais, representações profissionais (médicos, nutricionistas, enfermeiros e psicólogos, principalmente) e até movimentos especificamente organizados em nome da promoção do aleitamento materno, como o IBFAN (International Baby Foof Action Network). O estabelecimento de estatutos éticos normativos de comercialização de alimentos infantis produzidos como substitutivos do leite materno e, sobretudo, a definição de objetivos explícitos estabelecendo compromissos e metas universais a serem alcançadas na década de 90 (Reunião de Cúpula de Nova York) representam decisões políticas, doutrinárias e programáticas cruciais nesse sentido. 6

O Estado de Pernambuco assinala dois eventos marcantes e pioneiros nessa direção. O primeiro, refere-se a portaria da secretaria de saúde do estado, proibindo o uso do leite industrializado em maternidades públicas (Portaria 99, 03/12/1974). 18 O segundo, consiste no reconhecimento por parte das Nações Unidas do Instituto Materno Infantil de Pernambuco como o primeiro Hospital Amigo da Criança do Brasil, pela aplicação dos Dez Passos pelo Aleitamento Materno em suas dependências. ${ }^{19}$

Desta forma, a avaliação contida neste artigo, a partir de um estudo epidemiológico (a chamada II Pesquisa Estadual de Saúde e Nutrição), torna-se particularmente pertinente e relevante, ao enfocar a situação do aleitamento materno na segunda metade da década passada e avaliar fatores estatisticamente associados à prevalência e modalidades do processo, com ênfase nas características das mães e das famílias e do possível e esperado papel das ações de saúde na promoção ao aleitamento materno. A situação encontrada e as inferências analíticas obtidas dos resultados avaliados através do estudo de base populacional permitem estabelecer uma linha de referência consistente para se administrar as propostas de intervenções e em relação aos objetivos de novos estudos, considerando-se que, dentro de um ou dois anos, a pesquisa será repetida, com a mesma padronização metodológica.

Destaca-se, de início, a observação de que os resultados foram modestos, em relação às expectativas. As Nações Unidas 6 estabeleceram que os governos e sociedades de todo o mundo empreendessem esforços para que, no final da década, todas as mães tivessem condições subjetivas e objetivas para alimentarem seus filhos exclusivamente ao seio até os seis meses de idade, ou pelo menos, até os primeiros quatro meses. Por outro lado, o leite materno, como alimentação complementar, deveria ser mantido até os dois anos de idade.

Confrontados com esses objetivos quantitativos, os resultados obtidos no Estado de Pernambuco, já no fim de 1997 (cumpridos, portanto, 70\% da chamada "década da amamentação"), foram frustrantes. Aos seis meses (de fato, a meta referencial), apenas seis crianças $(0,95 \%)$ mamavam exclusivamente. Em relação à meta secundária (quatro meses), o alcance foi de $3,98 \%$, referente a 24 casos; são as crianças analisadas no estudo caso/controle. Finalmente, aos 18 meses, apenas uma das 603 crianças da amostra ainda recebia leite materno como parte de sua amamentação.

São, portanto, resultados muito distanciados das metas propostas, ou ainda, dos achados descritos em escala internacional e nacional, segundo relatos contemporâneos ao estudo aqui apontado. Assim, o Fundo das Nações Unidas para a Infância (UNICEF) descreve que, entre 1990 e 1996 cerca de $44 \%$ das crianças de todo o mundo eram alimentadas exclusivamente com leite materno dos 0 aos três meses de vida, freqüência que baixava para $38 \%$ na América Latina. No que se refere ao aleitamento continuado (dominante ou complementar) até os 23 meses, os valores eram, respectivamente, $50 \%$ e $22 \%$, no mundo e na América Latina. ${ }^{11}$

A defasagem seria também muito marcante em relação aos dados nacionais, Assim, Rea ${ }^{3}$ descreve que, entre 1986 a 1996 o aleitamento materno exclusivo teria aumentado sua freqüência aproximadamente 10 vezes. No final da década (1999), segundo o Ministério da Saúde, aos seis meses de idade $72,9 \%$ da crianças mamavam, sendo que $9,7 \%$ se alimentavam exclusivamente ao peito.14,15 Para o 
Nordeste, os valores eram praticamente equivalentes: respectivamente, $69,6 \%$ e 10,7\%.

Há, assim, uma grande discrepância com os resultados do nosso estudo, o que em grande parte poderia ser explicado pela aplicação dos rígidos critérios recomendados pela World Health Organization (WHO) 20-22 em relação ao aleitamento materno exclusivo, retirando dessa categoria os casos, muitos comuns no Nordeste e no estado de Pernambuco, do uso de água e de chás, para compensar a sede ou "limpar os intestinos". 23 No entanto, esse critério não explicaria a grande diferença no que se refere, simplesmente, à amamentação como ocorrência genérica (exclusiva ou não) aos seis meses, no Nordeste e no próprio estado de Pernambuco. É uma questão em aberto.

No que se refere à análise estatística dos fatores associados, ressalta-se a conveniência circunstancial de se utilizar o método caso/controle, dado o pequeno número de casos (apenas 24 em toda a amostra) que satisfaziam a condição "aleitamento materno exclusivo", entre as 603 crianças observadas no estudo de prevalência. Trata-se de uma solução um tanto sui generis, sob o ponto de vista conceitual e metodológico, desde que o caso passou a ser o evento desejado e não a condição patológica, como habitualmente acontece, Assim, ao invés de fatores de risco, buscou-se a freqüência de exposição a fatores de proteção; isso inverte também a lógica de estimativa dos riscos relativos (odds ratio).

Alguns resultados das análises podem parecer inesperados. São os exemplos dos partos cirúrgicos como fatores de proteção, quando, antes, as cesarianas funcionavam como handicaps para a promoção ao aleitamento materno.24 Menos surpreendente, mas ainda assim revelador de uma nova situação, seria o caso da escolaridade mais elevada ou da categoria de renda mais alta como condições favoráveis ao prolongamento do aleitamento materno exclusivo. Quinze anos antes, o aleitamento materno era uma prática mais disseminada entre famílias de baixa renda, mães analfabetas ou com baixa escolaridade. A alimentação artificial era um sinal de status, a amamentação ao seio uma prática dos mais pobres. A mesma lógica vale para o fato de que as mães que dispõem de seguro saúde ou que são assistidas em clínicas privadas, pagando, por conta própria, os honorários médicos, tendem a aderir à recomendação de amamentar seus filhos por períodos mais prolongados. Parece um contra-senso que a maior distância da residência ao serviço de saúde tenha aparecido como um fator de proteção ao aleitamento materno mais prolongado. Na realidade, o que deve ocorrer é que as mães sob melhores condições socioe- conômicas independem do fator distância para freqüentar seus serviços de saúde, liberando-se, portanto, da hipótese dessa condicionalidade. Em suma, ocorreu uma substancial mudança de atitude que favorece a prática da alimentação ao seio como um benefício em favor das famílias de melhores condições socioeconômicas, por critérios de renda, escolaridade, utilização de serviços privados de saúde, parto com assistência médica, maior número de consultas pré-natais, início mais precoce (primeiro trimestre) do acompanhamento pré-natal. Seria a diferenciação ao acesso qualitativo e quantitativo dos serviços e ações de saúde que mediariam as respostas em relação à amamentação. Em estudo efetuado no interior da Bahia, Vieira et al. ${ }^{25}$ observaram que, ao contrário de nossos resultados, os estratos de renda e de escolaridade mais baixos apresentaram valores mais elevados em termos de duração do aleitamento materno exclusivo. $\mathrm{O}$ fato de mamar após o nascimento e de não usar chupeta aparecem como fatores de proteção do aleitamento materno.

É interessante ressaltar que, praticamente em todas as situações, a orientação sobre aleitamento materno, como se revela na análise dos dados, figurava como uma norma de conduta educativa na assistência pré-natal. Comparados com resultados de outros estudos, concluise que a duração do aleitamento materno exclusivo e os fatores associados à sua evolução temporal não se comportam de forma homogênea, apresentando diferenciações marcantes. No caso de Pernambuco, se os resultados em favor do aleitamento materno não foram tão efetivos, em escala populacional, ou ainda, se se manifestaram de forma diferenciada em função da clivagem socioeconômica das famílias, convém ponderar que as classes sociais mais elevadas servem de modelo para o comportamento dos demais estratos: a classe média e, por último, os grupos de baixa renda. Foi assim com a histórica do desmame. Poderá ser assim com a reação em contrário. Os resultados da presente análise poderiam ser tomados como sinais preditivos desse novo e desejado comportamento? 


\section{Referências}

1. Tu P. The effects of breastfeeding and birth spacing on child survival in China. Stud Fam Plann 1989; 20: 332-42.

2. Almeida JAG. Amamentação: um híbrido natureza-cultura. Rio de Janeiro: Ed. Fiocruz; 2002.

3. Alves JGB, Figueira F. Doenças do adulto com raízes na infância. Recife: Bagaço; 1998.

4. WHO (World Health Organization). Global strategy for infant and young child feeding [on line]. Disponível em: http://www, who, int,nut/documents/gs<infant-feeding-texteng.pdf $>$ [2003 out 10].

5. Fuchs, SC, Victora, CG. Risk and prognostic factors for diarrheal disease in Brazilian infants: a special case-control design application. Cad Saúde Pública 2002; 18: 773-82.

6. UNICEF (Fundo das Nações Unidas para a Infância). Estratégia para melhorar a nutrição de crianças e mulheres nos países em desenvolvimento: um exame de políticas. New York; 1990.

7. Ministério da Saúde, OPAS (Organização Pan-Americana da Saúde). Guia alimentar para crianças menores de dois anos. Brasília (DF); 2005.

8. Winikof B, Castle M, Laukaran W. Feeding infants in four societies: causes and consequences of matters choices. Greenwood; 1989.

9. WHO (World Health Organization). Complementary feeding of young children in developing countries: a review of current scientific knowledge. Geneve; 1998.

10. Gartner LM, Black LS, Eaton AP, Lawrence RA, Naylor AJ, Neifert ME, O'Hare D, Schanler RJ. Breast feeding and the use of human milk. Pediatrics 1997; 6: 1035-9.

11. UNICEF (Fundo das Nações Unidas para a Infância). Situação mundial da infância: 1998. Brasília (DF); 1998.

12. UNICEF (Fundo das Nações Unidas para a Infância). Situação mundial da infância: 2002. Brasília (DF); 2002.

13. Rea MF. Reflexões sobre a amamentação no Brasil: de como passamos a 10 meses de duração. Cad Saúde Pública 2003; 19 (Supl.1): 37-45.

14. Ministério da Saúde. Prevalência de aleitamento materno, D.19. In: Indicadores de morbidade e fatores de risco. 2001. [on line]. Disponível em: <http://tabnet.datasus.gov.br/ cgi/idb.2001/d19.htm> [2002 set 7].

15. Ministério da Saúde. Prevalência de aleitamento materno exclusivo. D.20. In: Indicadores de morbidade e fatores de risco. 2001. [on line]. Disponível em: <http://Tabnet. datasus.gov.br /cgi/idb2001/d20.htm>. [2001 set 7].
16. Batista Filho M, Romani SAM. (Orgs.). Alimentação, nutrição e saúde no Estado de Pernambuco: espacialização e fatores sócio-econômicos. Recife: Instituto Materno Infantil de Pernambuco (IMIP), 2002.

17. Ebrahim GJ. Análise multivariada. In: Métodos de pesquisa, 2. Tradução: Figueira AC. Recife: Instituto Materno Infantil de Pernambuco, IMIP; 2002. p. 117-26.

18. Müller MO. Matador de bebês. Tradução: Instituto Materno Infantil de Pernambuco (IMIP). Recife; 2005.

19. Lamounier JÁ, Maranhão AGK, Araujo MFM. Iniciativa Hospital Amigo da Criança no Brasil. In: Rego JD. Aleitamento materno. São Paulo: Atheneu; 2001. p. 333-42.

20. WHO (World Health Organization). Indicators for assessing breask-feeding practices: reporto f na informal meeting. [on line]. Geneva; 1991. Disponível em: $<$ http://www.who.int/child-adolescent, health new publications/NUTRITION/WHO - CDD - SER - 91.14. pdf.> [2003 out 10].

21. WHO (World Health Organization). Exclusive breastfeeding, 2000. [on line]. Disponível em http://www.who.int/child-adolescent.health/exclusive breastfeeding for six months. Html [2002 jul 9].

22. WHO (World Health Organization). Global strategy for infant and young child feeding. 2003. 2003. [on line]. Disponível em http://www.who.int/nut/documents/gsinfant-feeding-text-eng.pdf>. [2003 out 10].

23. Coutinho SB. Aleitamento materno exclusivo: um estudo de intervenção randomizada na Zona da Mata Meridional de Pernambuco [tese doutorado]. Recife: Departamento de Nutrição, Centro de Ciências da Saúde, Universidade Federal de Pernambuco; 2003.

24. Weiderpass E, Barros FC, Victora CG, Tomasi E, Halpern R. Incidência e duração da amamentação conforme o tipo de parto: estudo longitudinal no Sul do Brasil. Rev Saúde Pública 1998; 32: 225-31.

25. Vieira GO, Guerra de Almeida JA, Silva LR, Cabral VA, Santana Netto PV. Fatores associados a aleitamento materno e desmame em Feira de Santana, Bahia. Rev Bras Saúde Matern Infant 2004; 4: 143-50.

Recebido em 25 de junho de 2005

Versão final apresentada em 19 de setembro de 2005

Aprovado em 1 de novembro de 2005. 